\title{
CLASS INVARIANTS FROM A NEW KIND OF WEBER-LIKE MODULAR EQUATION
}

\author{
WILLIAM B. HART
}

\begin{abstract}
A new technique is described for explicitly evaluating quotients of the Dedekind eta function at quadratic integers. These evaluations do not make use of complex approximations but are found by an entirely 'algebraic' method. They are obtained by means of specialising certain modular equations related to Weber's modular equations of 'irrational type'. The technique works for a large class of eta quotients evaluated at points in an imaginary quadratic field with discriminant $d \equiv 1(\bmod 8)$. In particular, this method does place any restriction on the class number of the associated imaginary quadratic number field.
\end{abstract}

\section{INTRODUCTION}

This paper is primarily concerned with the explicit computation of various class invariants coming from quotients of the Dedekind eta function, which is defined for all $\tau$ in the complex upper half plane by

$$
\eta(\tau)=q^{1 / 24} \prod_{n=1}^{\infty}\left(1-q^{n}\right)
$$

where $q=e^{2 \pi i \tau}$.

In particular we will express many of our eta quotients in terms of the three Weber functions:

$$
\mathfrak{f}(\tau)=\frac{e^{-\frac{\pi i}{24}} \eta\left(\frac{\tau+1}{2}\right)}{\eta(\tau)}, \quad \mathfrak{f}_{1}(\tau)=\frac{\eta\left(\frac{\tau}{2}\right)}{\eta(\tau)}, \quad \mathfrak{f}_{2}(\tau)=\sqrt{2} \frac{\eta(2 \tau)}{\eta(\tau)} .
$$

Such evaluations have a long history, and appear in the literature in a number of guises. Firstly we have Weber's own evaluations of these functions, in his Lehrbuch der Algebra [18]. He provides detailed tables listing values of the functions $\mathfrak{f}(\tau)$ and $\mathfrak{f}_{1}(\tau)$ at a large number of points of the form $\tau=\sqrt{-n}$, for natural numbers $n$.

Secondly, Ramanujan also provided numerous evaluations of related class invariants which he denoted $G_{n}$ and $g_{n}$, whose relationship to the Weber functions is given by

$$
\mathfrak{f}(\sqrt{-n})=2^{1 / 4} G_{n} \quad \text { and } \quad \mathfrak{f}_{1}(\sqrt{-n})=2^{1 / 4} g_{n}
$$

Some of Ramanujan's evaluations go beyond those given in the tables at the end of Weber's book. Many of his evaluations were later established by Watson [12], [13], [14], [15], [16] and [17], including some of those not appearing in Weber. The remaining evaluations were established by Berndt, Chan and Zhang, [2] and [3]. Further details on the history of this problem can also be found in Berndt's book $[1]$. 
The present work is concerned with the evaluation of an infinite class of values of the form

$$
\sqrt{\frac{a 2}{a 1}}\left|\frac{\eta\left(\frac{-b_{1}+\sqrt{d}}{2 a_{1}}\right)}{\eta\left(\frac{-b_{2}+\sqrt{d}}{2 a_{2}}\right)}\right|^{2},
$$

(or the cube of such values when $3 \mid d$ ), where $d$ is the discriminant of an imaginary quadratic number field, and $d=b_{i}^{2}-4 a_{i} c_{i} \equiv 1(\bmod 8)$, for rational integers $a_{i}, b_{i}, c_{i}$ for $i=1,2$.

This can be viewed as a generalisation of Weber's results. However, the true motivation for completing evaluations of this form, is the Kronecker limit formula. It has to do with the evaluation of Epstein zeta functions.

Given a binary quadratic form $(a, b, c):=Q(x, y)=a x^{2}+b x y+c y^{2}, \quad d=b^{2}-4 a c<$ 0 , the Epstein zeta function is defined to be

$$
Z(s)=\sum_{x, y=-\infty}^{\infty} \frac{1}{Q(x, y)^{s}}
$$

where the dash indicates that $(0,0)$ is omitted from the sum.

Then the Kronecker limit formula states

$$
\lim _{s \rightarrow 1} \sum_{[Q]} \sum_{x, y=-\infty}^{\infty} \frac{\chi([Q])}{Q(x, y)^{s}}=\frac{-8 \pi}{\sqrt{|d|}} \sum_{[Q]} \chi([Q]) \log \left(a^{-1 / 4}\left|\eta\left(\frac{b+\sqrt{d}}{2 a}\right)\right|\right) .
$$

For more details on quotients of the eta function and their relationship to the Kronecker limit formula, Chowla-Selberg formula, special values of $L$-series and explicit class field theory, see the author's thesis [10].

We note that recently, Muzaffar and Williams [11] announced a method for evaluating the eta function itself at quadratic integers. Their method makes use of the theory of binary quadratic forms, and provides evaluations in terms of certain character sums over functions which they define in their paper.

The method presented in our paper, in distinction, makes use of the theory of modular equations and provides evaluations of class invariants by giving minimal polynomials for them.

As noted in the author's thesis [10], prior techniques in the literature for providing explicit evaluations relied on various properties of the class group of the associated imaginary quadratic number field - usually that the class group be 2-torsion. As pointed out in [2],

The algebraic nature of the class invariants depends upon properties such as the class number of $\mathbb{Q}(\sqrt{-n})$, the genus structure, the number of classes per genus, and the genus characters...

The method we present here provides a way of evaluating an infinite number of class invariants without restrictions on the specific form of the class group. The only restriction we place on the associated imaginary quadratic number field, is that the discriminant be congruent to 1 modulo 8. Our method does not necessarily evaluate all eta quotients associated with a given discriminant, but it does evaluate a significant subset of them.

We provide various explicit examples of our method, demonstrating the above features. Note that the case of providing evaluations where the class number is three 
has already been dealt with by Chapman and van der Poorten [5], by class field theoretic methods. Therefore in this paper, we will illustrate our method by examples where the class number is five and higher.

Note that although we call our evaluations class invariants, we have been unable to find a complete result in the literature stating when eta quotients of the form outlined above, are actually class invariants.

Class invariants, as Weber defined them, are essentially values, at points $\omega$ in some imaginary quadratic number field $K$, of modular functions $f(\tau)$, with the following special property: the functions $f(\tau)$ themselves lie in an extension field of the function field $\mathbb{C}(j(\tau)$ ) (where $j$ is the usual modular invariant function) but the values $f(\omega)$ actually lie in the field $K(j(\omega))$.

Equivalently, a class invariant is a special value $f(\omega)$ which lies in the Hilbert class field of $K$. One can extend the definition to include values $f(\omega)$ lying in a ring class field of $K$, where $\omega$ is taken in an appropriate quadratic order of $K$.

As mentioned, there is no completely general statement in the literature about values of the form

$$
\left|\frac{\eta(\omega / n)}{\eta(\omega)}\right|^{k},
$$

given the quadratic order in which $\omega$ lies and a rational integer $k$ (note the evaluations above can be reduced to this form by making modular transformations of the values plugged into the eta function). In fact, one would ideally like to know how to normalise values of this form which do turn out to be class invariants, so that they become units in the corresponding ring class fields of $\mathbb{Q}(\sqrt{d})$.

For the case of Weber functions $(n=2)$, Weber does make some statements along these lines in his Lehrbuch [18], which were completely proved by Birch [4]. Some more general results have been provided by Hajir [7]. Also, Alice Gee [6] has some statements about class invariants coming from various 'level' three and five variants of the Weber functions $(n=3,5)$. Further examples can be found in the author's thesis [10]. However none of these statements approach full generality, even in the case of Weber functions.

In this paper, we will abuse terminology somewhat and call all our eta quotients, class invariants, leaving the task of showing that they actually are, for another time. It might be noted however, that in any particular case, it is always possible to determine whether an evaluation is a class invariant or a unit, since we are providing a minimal polynomial for the value. For, basic arguments show that our value must lie in an extension field of the appropriate ring class field and thus a simple check on the degree of the minimal polynomial is sufficient to determine that the value actually lies in the ring class field. Of course, it is trivial to show that the values are units, given their minimal polynomials.

The general method employed in this paper is to specialise certain new modular equations involving the Weber functions. These modular equations are described in our recent work [9], where explicit examples of these equations are also computed. To begin this paper, we felt that it would be of value to the modern reader to provide a summary of the methods of Weber in computing class invariants via modular equations. This is particularly relevant since his methods inspired our own to some degree.

\section{Weber's Own Evaluations}

Weber is, with few exceptions, interested in evaluating one of his functions $\mathfrak{f}$ or $\mathfrak{f}_{1}$ at $\sqrt{-m}$ for some natural number $m$. 
In $\S 128$ of his Lehrbuch [18] he uses previously calculated values of the function $\gamma_{2}(\omega)=\sqrt[3]{j(\omega)}$ and the equation

$$
u^{3}-\gamma_{2}(\omega) u-16=0,
$$

which has roots $\mathfrak{f}(\omega)^{8},-\mathfrak{f}_{1}(\omega)^{8}$ and $-\mathfrak{f}_{2}(\omega)^{8}$, to calculate minimal polynomials for $\mathfrak{f}(\omega)$ at certain values of $\omega$. However this method only works for him in situations where he can explicitly calculate the value $\gamma_{2}(\omega)$ and where it has a 'nice' value, such as a rational integer. In fact this happens for $\omega=\sqrt{-p}$, where $p$ is a prime discriminant (or $p=1$ ) corresponding to an imaginary quadratic number field with class number one. Thus Weber calculates minimal polynomials for $\mathfrak{f}(\sqrt{-p})$ when $p=1,3,7,11,19,43,67$ and 163 . He also calculates $\mathfrak{f}_{1}(\sqrt{-2})$ in a similar way.

In $\S 129$, by using transformations of second order which are identities derived from the well known identity

$$
\mathfrak{f}_{1}(2 \omega) \mathfrak{f}_{2}(\omega)=\sqrt{2},
$$

Weber is able to use the above results to compute values of $\mathfrak{f}_{1}(\sqrt{-m})$ for the composite values $m=4,8,12,16,28$ and 32 .

By $§ 130$ Weber has realised that he can use both Schläfli modular equations and his modular equations of 'irrational kind' to obtain evaluations.

A Schläfli modular equation provides a polynomial relation between $u=\mathfrak{f}(\omega)$ and $v=\mathfrak{f}(n \omega)$ or between $u=\mathfrak{f}_{1}(\omega)$ and $v=\mathfrak{f}_{1}(n \omega)$, (usually where $n$ is prime, in Weber's case). These modular equations can be exploited by setting $\omega=\sqrt{-m}$ for some integer $m$, providing a polynomial relation between $\mathfrak{f}(\sqrt{-m})$ and $\mathfrak{f}\left(\sqrt{-n^{2} m}\right)$, or the equivalent thing for $\mathfrak{f}_{1}$.

This method is particularly useful for cases where the value of $\mathfrak{f}(\sqrt{-m})\left(\operatorname{or}_{1}(\sqrt{-m})\right)$ is known and is a nice value such as the square root of an integer. This is certainly the case for $m=1,2,3,4$ or 7 . With this technique Weber is able to compute the values $\mathfrak{f}\left(\sqrt{-n^{2} m}\right)$ where $n^{2} m=9,25,27,49,63,75$ and 175 , and $\mathfrak{f}_{1}\left(\sqrt{-n^{2} m}\right)$ where $n^{2} m=18,36,50$ and 100 .

There are of course higher values of $n^{2} m$ for which this technique works, but one sees the limited applicability of it from Weber's point of view, due to its reliance on a known simple value being given for $\mathfrak{f}(\sqrt{-m})$.

Next Weber makes use of the fact that $\sqrt{-m} / m=-1 / \sqrt{-m}$. It is easy to see that if one sets $\omega=\sqrt{-m} / m$ then

$$
\mathfrak{f}(\omega)=\mathfrak{f}(-1 / \sqrt{-m})=\mathfrak{f}(\sqrt{-m})=\mathfrak{f}(m \omega) .
$$

Using this information, a Schläfli modular equation of degree $m$ involving $\mathfrak{f}(\omega)$ and $\mathfrak{f}(m \omega)$ becomes a minimum polynomial for $\mathfrak{f}(\sqrt{-m})$. Using this method, Weber obtains the additional evaluations $\mathfrak{f}(\sqrt{-m})$ for $m=5,13$ and 17 .

Next Weber takes $\omega$ to be a root of $2 x^{2}+2 r x+n$ where $n$ is odd and $r$ any integer. In fact he takes $\omega=(-r+\sqrt{-m}) / 2$ where $m=2 n-r^{2}$. Of particular importance is the fact that $-n / \omega=r+\sqrt{-m}$. The idea is to consider the modular equation of degree $n$ relating $\mathfrak{f}_{2}(\omega)$ and $\mathfrak{f}_{2}(\omega / n)$. A simple calculation then shows that these have the values $\exp (-r \pi i / 24) \sqrt{2} / x$ and $\exp (-r \pi i / 24) x$ respectively, where $x=\mathfrak{f}_{1}(\sqrt{-m})$ or $x=\mathfrak{f}(\sqrt{-m})$, depending on whether $r$ is odd or even.

Weber gives a bewildering array of values $m$ and $n$ for which this method works. In particular, he is able to add to his list of evaluations $f(\sqrt{-13})$ and $\mathfrak{f}_{1}(\sqrt{-m})$ for $m=10,22$ and 26 .

Next, Weber sets $\omega$ to be the root $(-r+\sqrt{-m}) /(2 n)$ of $2 n x^{2}+2 r x+n$ where $m=2 n^{2}-r^{2}$. This time he considers the values $\mathfrak{f}_{2}(\omega), \mathfrak{f}_{2}(\omega / n)$ and $\mathfrak{f}_{2}(n \omega)$. Again 
depending on whether $r$ is odd or even, the last two of these can both be expressed in terms of $x=\mathfrak{f}_{1}(\sqrt{-m})$ or $x=\mathfrak{f}(\sqrt{-m})$. Combining two Schläfli modular equations of degree $n$ in an obvious way, one can then eliminate $\mathfrak{f}_{2}(\omega)$, leaving only a relation between $\mathfrak{f}_{2}(\omega / n)$ and $\mathfrak{f}_{2}(n \omega)$. This leaves, of course, an equation involving only the expression $x$.

Weber uses this technique to evaluate $\mathfrak{f}(\sqrt{-41})$.

Now comes Weber's $\S 131$ where he makes use of the modular equations of irrational form. These are modular equations that involve all three of his Weber functions $\mathfrak{f}$, $\mathfrak{f}_{1}$ and $\mathfrak{f}_{2}$.

For example, consider Weber's modular equations of degree $n \equiv 7(\bmod 8)$ of this form. They are polynomial relations between functions of the form

$$
2 A=\mathfrak{f}(\omega) \mathfrak{f}(n \omega)+(-1)^{\frac{n+1}{8}}\left(\mathfrak{f}_{1}(\omega) \mathfrak{f}_{1}(n \omega)+\mathfrak{f}_{2}(\omega) \mathfrak{f}_{2}(n \omega)\right)
$$

and

$$
B=\frac{2}{\mathfrak{f}_{1}(\omega) \mathfrak{f}_{1}(n \omega)}+\frac{2}{\mathfrak{f}_{2}(\omega) \mathfrak{f}_{2}(n \omega)}+(-1)^{\frac{n+1}{8}} \frac{2}{\mathfrak{f}(\omega) \mathfrak{f}(n \omega)} .
$$

One then sets $\omega=-1 / \sqrt{-n}$, so that $n \omega=\sqrt{-n}$. Then set $\sqrt{2} x=\mathfrak{f}(\sqrt{-n})$. Note that $\mathfrak{f}_{1}(\omega) \mathfrak{f}_{1}(n \omega)$ becomes $\mathfrak{f}_{2}(\sqrt{-n}) \mathfrak{f}_{1}(\sqrt{-n})=\sqrt{2} / \mathfrak{f}(\sqrt{-n})=1 / x$, etc. In fact we have

$$
A=x^{2}+(-1)^{\frac{m+1}{8}} / x \text { and } B=4 x+(-1)^{\frac{m+1}{8}} / x^{2},
$$

(where we correct a typographical error at this point in Weber). In other words, the modular equation of irrational form, being a polynomial relationship between the functions $A$ and $B$, induces a polynomial equation in the single value $x$, which, when factored, provides a minimal polynomial for the value $x=\mathfrak{f}(\sqrt{-n}) / \sqrt{2}$.

This method allows Weber to obtain new evaluations for $\mathfrak{f}(\sqrt{-n})$ where $n=23,31$, 47 and 71 .

We mention one final method of Weber's, involving his modular equations of irrational form. His idea is somewhat limited in that it only applies to a modular equation where the function $B$ does not appear.

In the expression for $A$, he picks the value of $\omega$ in such a way that the term involving $\mathfrak{f}_{2}(\omega) \mathfrak{f}_{2}(\omega / n)$ becomes a particularly simple constant. The remaining terms involving $\mathfrak{f}$ and $\mathfrak{f}_{1}$ he evaluates by making use of the following identity of degree 2 which he also derives in $\S 131$ :

$$
\mathfrak{f}(\omega)^{4} \mathfrak{f}(2 \omega)^{4}+\mathfrak{f}_{1}(\omega)^{4} \mathfrak{f}_{2}(2 \omega)^{4}=\mathfrak{f}_{2}(\omega)^{6}+8 / \mathfrak{f}_{2}(\omega)^{6} .
$$

The way he makes use of this identity is to carefully choose the value $\omega$. He sets $\omega=(-r+\sqrt{-m}) / 2$, a root of $2 x^{2}+2 r x+n$, for some integer $r$ and $m=2 n-r^{2}$. The crucial benefit of this is that $2 \omega=-n / \omega-2 r$, making the terms on the left hand side of the degree two identity equal to some power of the terms involving $\mathfrak{f}$ and $\mathfrak{f}_{1}$ in his modular equation of irrational form.

After taking the appropriate power of his modular equation and substituting in the degree two identity, Weber ends up with an expression containing only the value $\mathfrak{f}_{2}(\omega)$ and some known constants. But depending on whether or not $r$ is even, $\mathfrak{f}_{2}(\omega)$ turns out to be (up to some simple constant factors) either $\mathfrak{f}(\sqrt{-m})$ or $\mathfrak{f}_{1}(\sqrt{-m})$. Thus a minimal polynomial for one of these two values is obtained.

Weber is able to use this process to obtain an evaluation of $\mathfrak{f}_{1}(\sqrt{-46})$, since he can set $m=46, n=23$ and $r=0$.

A final method which Weber gives in $\S 131$ makes use of his theory of modular equations of composite degree. It only allows Weber to add to his list an evaluation for $\mathfrak{f}(\sqrt{-39})$. 


\section{New Evaluations Using Weber Modular Equations}

Before moving on to the general method presented in this paper, we demonstrate that a number of evaluations of the kind we are interested in can be completed using the Weber modular equations of irrational form. Although this technique is somewhat limited, it is nonetheless interesting in its own right, and is this method which led us to the more general one explained later.

We begin with evaluations where the class number of the associated imaginary quadratic number field is five.

2.1. Discriminant $\mathbf{- 4 7}$. We will evaluate the eta quotients

$$
m_{1}=\left|\frac{\eta\left(\tau_{2}\right)}{\eta\left(\tau_{1}\right)}\right|^{2}=\left|\mathfrak{f}_{1}\left(\tau_{1}\right)\right|^{2} \quad \text { and } \quad m_{2}=\sqrt{\frac{3}{2}}\left|\frac{\eta\left(\tau_{2}\right)}{\eta\left(\tau_{3}\right)}\right|^{2},
$$

where

$$
\tau_{1}=\frac{1+\sqrt{-47}}{2}, \quad \tau_{2}=\frac{1+\sqrt{-47}}{4} \text { and } \tau_{3}=\frac{1+\sqrt{-47}}{6} .
$$

These values are of interest because a complete set of reduced binary quadratic forms for discriminant -47 is given by $(1,1,12),(2, \pm 1,6)$ and $(3, \pm 1,4)$ and three of these have roots $\tau_{1}, \tau_{2}$ and $\tau_{3}$ respectively. We do not need to consider separately roots of the remaining two reduced forms, since no new eta values would be obtained, as follows immediately from the following well known identity

$$
\eta\left(\frac{-b+\sqrt{d}}{2 a}\right)=\overline{\left(\frac{b+\sqrt{d}}{2 a}\right)},
$$

with $a, b$ and $-d \in \mathbb{N}$, (after taking complex absolute values of both sides).

Clearly any other eta quotient involving the values $\tau_{1}, \tau_{2}$ and $\tau_{3}$ can be obtained from combining the values $m_{1}$ and $m_{2}$. In fact, any eta quotient involving roots of binary quadratic forms of discriminant -47 can be reduced by means of modular transformations of the given arguments, to evaluations of the eta quotients $m_{1}$ and $m_{2}$.

In the following, we make repeated use of the standard modular transformations of the three Weber functions, without comment.

Firstly, we make use of Weber's evaluation of $x=|\mathfrak{f}(\sqrt{-47})| / \sqrt{2}$. He states in the tables at the back of his Lehrbuch [18] that $x$ satisfies $x^{5}-x^{3}-2 x^{2}-2 x-1=0$.

But we notice that

$$
\left|\mathfrak{f}_{2}\left(\tau_{1}\right)\right|=\sqrt{2} /\left|\mathfrak{f}_{1}(1+\sqrt{-47})\right|=\sqrt{2} /|\mathfrak{f}(\sqrt{-47})|
$$

Next we notice that

$$
\left|\mathfrak{f}\left(\frac{1+\sqrt{-47}}{2}\right)\right|=\left|\mathfrak{f}_{1}\left(\frac{-1+\sqrt{-47}}{2}\right)\right|=\left|\mathfrak{f}_{1}\left(\tau_{1}\right)\right|,
$$

the second equality following from (5).

But now from the well known identity

$$
\mathfrak{f} \mathfrak{f}_{1} \mathfrak{f}_{2}=\sqrt{2}
$$

we calculate that

$$
\left|\mathfrak{f}_{1}\left(\tau_{1}\right)\right|^{2}=\sqrt{2} /\left|\mathfrak{f}_{2}\left(\tau_{1}\right)\right|=|\mathfrak{f}(\sqrt{-47})| .
$$

Thus we have that $m_{1} / \sqrt{2}=\left|\mathfrak{f}_{1}\left(\tau_{1}\right)\right|^{2} / \sqrt{2}$ also satisfies $x^{5}-x^{3}-2 x^{2}-2 x-1=0$.

Next we notice that

$$
\left|\mathfrak{f}_{2}\left(\tau_{2}\right)\right|=\sqrt{2} /\left|\mathfrak{f}_{1}\left(\tau_{1}\right)\right|
$$


by (4).

Thus from what we have already calculated

$$
\left|\mathfrak{f}_{2}\left(\tau_{2}\right)\right|^{2} / \sqrt{2} \text { satisfies } y^{5}+2 y^{4}+2 y^{3}+y^{2}-1=0 .
$$

We will now relate this value to $\left|\mathfrak{f}_{1}\left(\tau_{2}\right)\right|^{2} / \sqrt{2}$ by means of a modular equation of degree three for Weber functions. This is done as follows. Firstly we find

$$
\mathfrak{f}_{1}\left(\frac{-1+\sqrt{-47}}{4}\right)=\mathfrak{f}_{2}\left(\frac{1+\sqrt{-47}}{12}\right),
$$

and

We also note that

$$
\mathfrak{f}_{2}\left(\frac{-1+\sqrt{-47}}{4}\right)=\mathfrak{f}_{1}\left(\frac{1+\sqrt{-47}}{12}\right) \text {. }
$$

$$
\mathfrak{f}\left(\frac{-1+\sqrt{-47}}{4}\right)=\mathfrak{f}\left(\frac{1+\sqrt{-47}}{12}\right) .
$$

The type of modular equation which we will use is that of irrational form spoken of by Weber in $\S 75$ of [18]. The degree three equation of this kind is

$$
\mathfrak{f}(\omega)^{2} \mathfrak{f}(3 \omega)^{2}=\mathfrak{f}_{1}(\omega)^{2} \mathfrak{f}_{1}(3 \omega)^{2}+\mathfrak{f}_{2}(\omega)^{2} \mathfrak{f}_{2}(3 \omega)^{2} .
$$

If we substitute in the value $\omega=\frac{1+\sqrt{-47}}{12}$ then this becomes

$$
|\mathfrak{f}(3 \omega)|^{4}=\overline{\mathfrak{f}_{2}}(3 \omega)^{2} \mathfrak{f}_{1}(3 \omega)^{2}+\overline{\mathfrak{f}_{1}}(3 \omega)^{2} \mathfrak{f}_{2}(3 \omega)^{2},
$$

making use of (5).

We wish to do away with the asymmetry on the right hand side of this equation. Calling terms 'symmetric', which only involve values multiplied by their complex conjugates, we attempt to rearrange this equation so that it only involves symmetric terms. This is done by repeatedly squaring the equation and placing all the symmetric terms on one side, and the remaining terms on the other.

After doing this twice (always replacing $\left|\mathfrak{f}_{1}(3 \omega) \mathfrak{f}_{2}(3 \omega)\right|$ with $\sqrt{2} /|\mathfrak{f}(3 \omega)|$ wherever it occurs) we obtain

$$
\begin{gathered}
|\mathfrak{f}(3 \omega)|^{16}+32 /|\mathfrak{f}(3 \omega)|^{8}-16|\mathfrak{f}(3 \omega)|^{4}=\mathfrak{f}_{1}(3 \omega)^{8} \overline{\mathfrak{f}_{2}}(3 \omega)^{8}+\mathfrak{f}_{2}(3 \omega)^{8} \overline{\mathfrak{f}_{1}}(3 \omega)^{8} \\
=\mathfrak{f}_{1}(3 \omega)^{8}\left(\overline{\mathfrak{f}}(3 \omega)^{8}-\overline{\mathfrak{f}_{1}}(3 \omega)^{8}\right)+\mathfrak{f}_{2}(3 \omega)^{8}\left(\overline{\mathfrak{f}}(3 \omega)^{8}-\overline{\mathfrak{f}_{2}}(3 \omega)^{8}\right) \\
=-\left|\mathfrak{f}_{1}(3 \omega)\right|^{16}-\left|\mathfrak{f}_{2}(3 \omega)\right|^{16}+|\mathfrak{f}(3 \omega)|^{16}
\end{gathered}
$$

In actual fact, the same argument shows in general that

$$
16|\mathfrak{f}(\tau)|^{4}-32 /|\mathfrak{f}(\tau)|^{8}=\left|\mathfrak{f}_{1}(\tau)\right|^{16}+\left|\mathfrak{f}_{2}(\tau)\right|^{16}
$$

when we have $\tau=\frac{b+\sqrt{d}}{2 a}$ and $-1 / \tau=\frac{-b+\sqrt{d}}{6 a}$. This is equivalent to the condition that $\tau$ be a root of a binary quadratic form $(a, b, c)$ with $c=3 a$, which certainly holds for our value $\tau=\tau_{2}$.

Then letting $l=|\mathfrak{f}(\tau)|^{2}, m=\left|\mathfrak{f}_{1}(\tau)\right|^{2} / \sqrt{2}$ and $n=\left|\mathfrak{f}_{2}(\tau)\right|^{2} / \sqrt{2}$ so that $l m n=1$ we see that

$$
16 /(m n)^{2}-64(m n)^{4}=16 m^{8}+16 n^{8},
$$

which after rearranging and taking the square root becomes

$$
1 / m n=m^{4}+n^{4},
$$

or the elegant

$$
m^{5} n+n^{5} m=1
$$


But for $\tau=\tau_{2}$, the value $n$ is precisely the value given in the equation (7) above. Therefore we only need to solve the current equation in order to obtain the eta evaluation for $m$.

Initially we solved this equation by noting numerically (with a computer) that $l=n+1$. Thus from $l m n=1$ we were able to find a minimal polynomial for $m$. Suprisingly this numerical trick worked for many evaluations. However there is of course a much simpler way of doing it.

Using a computer alebra package, one simply takes the resultant of the equation (10) and the minimal polynomial for $n$ and eliminates $n$. One of the factors of the resulting expression is the minimal polynomial for $m$, which can be identified by substituting in the complex value of $m$ and checking which of the factors it is a root of.

Of course if one knew a priori the degree of the required minimal polynomial, one could more quickly identify it.

Either way, we find that $m$ has minimum polynomial $x^{5}+x^{4}+x^{3}-x^{2}-2 x-1$.

Now we prove that $m=1 / m_{2}$. We note

$$
\begin{aligned}
& m_{2}=\sqrt{\frac{3}{2}}\left|\frac{\eta((1+\sqrt{-47}) / 4)}{\eta((1+\sqrt{-47}) / 6)}\right|^{2} \\
& =\sqrt{\frac{3}{2}}\left|\frac{\eta((1+\sqrt{-47}) / 4)}{\sqrt{-i(-1+\sqrt{-47}) / 8} \eta((-1+\sqrt{-47}) / 8)}\right|^{2}=\sqrt{2} /\left|\mathfrak{f}_{1}\left(\tau_{2}\right)\right|^{2},
\end{aligned}
$$

which is the inverse of the value $m$ that we have calculated above.

We can continue from this evaluation and obtain further interesting ones.

Firstly we see that

$$
\left|\mathfrak{f}_{1}\left(\frac{1+\sqrt{-47}}{4}\right)\right|=\left|\mathfrak{f}_{2}\left(\frac{-1+\sqrt{-47}}{12}\right)\right|=\sqrt{2} /\left|\mathfrak{f}_{1}\left(\frac{-1+\sqrt{-47}}{6}\right)\right| .
$$

Thus $\left|\mathfrak{f}_{1}\left(\frac{1+\sqrt{-47}}{6}\right)\right|^{2} / \sqrt{2}$ satisfies $z^{5}+2 z^{4}+z^{3}-z^{2}-z-1=0$.

Now we do an interesting side calculation. We note

$$
\begin{aligned}
& \left|\mathfrak{f}\left(\frac{1+\sqrt{-47}}{6}\right)\right|=\left|\mathfrak{f}_{1}\left(\frac{-5+\sqrt{-47}}{6}\right)\right|=\sqrt{2} /\left|\mathfrak{f}_{2}\left(\frac{-5+\sqrt{-47}}{12}\right)\right| \\
= & \sqrt{2} /\left|\mathfrak{f}_{1}\left(\frac{5+\sqrt{-47}}{6}\right)\right|=\sqrt{2} /\left|\mathfrak{f}\left(\frac{-1+\sqrt{-47}}{6}\right)\right|=\sqrt{2} /\left|\mathfrak{f}\left(\frac{1+\sqrt{-47}}{6}\right)\right| .
\end{aligned}
$$

Thus, $\left|\mathfrak{f}\left(\frac{1+\sqrt{-47}}{6}\right)\right|^{2}=\sqrt{2}$. Combining this with (4) we find

$$
\left|\mathfrak{f}_{2}\left(\frac{1+\sqrt{-47}}{6}\right)\right|^{2}=\sqrt{2} /\left|\mathfrak{f}_{1}\left(\frac{1+\sqrt{-47}}{6}\right)\right|^{2},
$$

and therefore these values satisfy $x^{5}+x^{4}+x^{3}-x^{2}-2 x-1=0$.

However since $\left|\mathfrak{f}_{2}\left(\frac{1+\sqrt{-47}}{6}\right)\right|=\sqrt{2} /\left|\mathfrak{f}_{1}\left(\frac{1+\sqrt{-47}}{3}\right)\right|$ we have that

$$
\left|\mathfrak{f}_{1}\left(\frac{1+\sqrt{-47}}{3}\right)\right|^{2} / 2 \text { satisfies } z^{5}+2 z^{4}+z^{3}-z^{2}-z-1=0 .
$$

We summarise the eta evaluations that we have completed for the discriminant $d=-47$ in the above table below. 


\begin{tabular}{|c|c|}
\hline Eta Evaluations $(d=-47)$ & Minimum Polynomial \\
\hline$=\left|\mathfrak{f}_{1}\left(\frac{1+\sqrt{-47}}{2}\right)\right|^{2} / \sqrt{2}=\sqrt{2} /\left|\mathfrak{f}_{2}\left(\frac{1+\sqrt{-47}}{4}\right)\right|^{2}$ & $x^{5}-x^{3}-2 x^{2}-2 x-1$ \\
\hline$\left|\mathfrak{f}_{1}\left(\frac{1+\sqrt{-47}}{4}\right)\right|^{2} / \sqrt{2}=\sqrt{2} /\left|\mathfrak{f}_{1}\left(\frac{1+\sqrt{-47}}{6}\right)\right|^{2}$ & $x^{5}+x^{4}+x^{3}-x^{2}-2 x-1$ \\
$=\left|\mathfrak{f}_{2}\left(\frac{1+\sqrt{-47}}{6}\right)\right|^{2}=2 /\left|\mathfrak{f}_{1}\left(\frac{1+\sqrt{-47}}{3}\right)\right|^{2}$ & $x^{2}-2$ \\
\hline$\left|\mathfrak{f}\left(\frac{1+\sqrt{-47}}{6}\right)\right|^{2}$ & \\
\hline
\end{tabular}

Table 2.1

2.2. Discriminant -79. We move on to the next discriminant of class number five which is $d=-79$. Here we wish to evaluate the quantities

$$
m_{1}=\sqrt{2} /\left|\mathfrak{f}_{1}\left(\frac{1+\sqrt{-79}}{2}\right)\right|^{2} \text { and } m_{2}=\sqrt{2} /\left|\mathfrak{f}_{1}\left(\frac{1+\sqrt{-79}}{4}\right)\right|^{2} \text {. }
$$

These come from reduced forms of discriminant -79 with roots $\tau_{1}=\frac{1+\sqrt{-79}}{2}, \tau_{1}=$ $\frac{1+\sqrt{-79}}{4}$ and $\tau_{3}=\frac{1+\sqrt{-79}}{8}$.

Unfortunately Weber does not evaluate $|\mathfrak{f}(\sqrt{-79})| / \sqrt{2}$, however Ramanujan has essentially done it. According to the table of class invariants of 34.2 of [1] it satisfies the equation $x^{5}-3 x^{4}+2 x^{3}-x^{2}+x-1=0$.

Now we note that

$$
\left|\mathfrak{f}_{2}\left(\tau_{1}\right)\right|=\sqrt{2} /\left|\mathfrak{f}_{1}(1+\sqrt{-79})\right|=\sqrt{2} /|\mathfrak{f}(\sqrt{-79})| .
$$

Also we have

$$
\left|\mathfrak{f}\left(\tau_{1}\right)\right|=\left|\mathfrak{f}_{1}\left(\frac{-1+\sqrt{-79}}{2}\right)\right|=\left|\mathfrak{f}_{1}\left(\tau_{1}\right)\right|
$$

Therefore from (4) we find that

$$
\left|\mathfrak{f}_{1}\left(\tau_{1}\right)\right|^{2} / \sqrt{2} \text { satisfies } x^{5}-3 x^{4}+2 x^{3}-x^{2}+x-1=0 .
$$

Also we have

Thus in particular

$$
\left|\mathfrak{f}_{2}\left(\tau_{2}\right)\right|=\sqrt{2} /\left|\mathfrak{f}_{1}\left(\tau_{1}\right)\right|
$$

$$
\left|\mathfrak{f}_{2}\left(\tau_{2}\right)\right|^{2} / \sqrt{2} \text { satisfies } y^{5}-y^{4}+y^{3}-2 y^{2}+3 y-1=0 .
$$

We now relate this value to another via a modular equation of degree 5 .

Firstly we have

and

$$
\mathfrak{f}_{2}\left(\frac{-1+\sqrt{-79}}{4}\right)=\mathfrak{f}_{1}\left(\frac{1+\sqrt{-79}}{20}\right)
$$

$$
\mathfrak{f}_{1}\left(\frac{-1+\sqrt{-79}}{4}\right)=\mathfrak{f}_{2}\left(\frac{1+\sqrt{-79}}{20}\right)
$$

and

$$
\mathfrak{f}\left(\frac{-1+\sqrt{-79}}{4}\right)=\mathfrak{f}\left(\frac{1+\sqrt{-79}}{20}\right) \text {. }
$$

We employ Weber's modular equation of irrational form of degree 5. This is given in $\S 75$ of [18] as

$$
8=\mathfrak{f}(\omega)^{4} \mathfrak{f}(5 \omega)^{4}-\mathfrak{f}_{1}(\omega)^{4} \mathfrak{f}_{1}(5 \omega)^{4}-\mathfrak{f}_{2}(\omega)^{4} \mathfrak{f}_{2}(5 \omega)^{4} .
$$




\begin{tabular}{|c|c|}
\hline Eta Evaluations $(d=-79)$ & Minimum Polynomial \\
\hline$|\mathfrak{f}(\sqrt{-79})| / \sqrt{2}=1 /\left|\mathfrak{f}_{2}\left(\frac{1+\sqrt{-79}}{2}\right)\right|$ & $x^{5}-3 x^{4}+2 x^{3}-x^{2}+x-1$ \\
$=\left|\mathfrak{f}_{1}\left(\frac{1+\sqrt{-79}}{2}\right)\right|^{2} / \sqrt{2}=\sqrt{2} /\left|\mathfrak{f}_{2}\left(\frac{1+\sqrt{-79}}{4}\right)\right|^{2}$ & \\
\hline$\left|\mathfrak{f}_{1}\left(\frac{1+\sqrt{-79}}{4}\right)\right|^{2} / \sqrt{2}=\sqrt{2} /\left|\mathfrak{f}_{2}\left(\frac{1+\sqrt{-79}}{8}\right)\right|^{2}$ & $x^{5}+2 x^{4}-3 x^{2}-2 x-1$ \\
$=\left|\mathfrak{f}_{1}\left(\frac{1+\sqrt{-79}}{8}\right)\right|^{2}$ & $x^{2}-2$ \\
\hline$\left|\mathfrak{f}\left(\frac{1+\sqrt{-79}}{8}\right)\right|^{2}$ & \\
\hline
\end{tabular}

Table 2.2

Once we substitute the particular value $\omega=\frac{1+\sqrt{-79}}{20}$ this can be written

$$
8=|\mathfrak{f}(5 \omega)|^{8}-\overline{\mathfrak{f}_{2}}(5 \omega)^{4} \mathfrak{f}_{1}(5 \omega)^{4}-\overline{\mathfrak{f}_{1}}(5 \omega)^{4} \mathfrak{f}_{2}(5 \omega)^{4} .
$$

We apply the same technique as we did for $d=-47$, putting the asymmetric parts to one side and squaring. Doing this just once we obtain

$$
\begin{gathered}
|\mathfrak{f}(5 \omega)|^{16}-16|\mathfrak{f}(5 \omega)|^{8}+64=\overline{\mathfrak{f}_{2}}(5 \omega)^{8} \mathfrak{f}_{1}(5 \omega)^{8}+\overline{\mathfrak{f}_{1}}(5 \omega)^{8} \mathfrak{f}_{2}(5 \omega)^{8}+2\left|\mathfrak{f}_{1}(5 \omega) \mathfrak{f}_{2}(5 \omega)\right|^{8} \\
=\overline{\mathfrak{f}_{2}}(5 \omega)^{8}\left(\mathfrak{f}(5 \omega)^{8}-\mathfrak{f}_{2}(5 \omega)^{8}\right)+\overline{\mathfrak{f}_{1}}(5 \omega)^{8}\left(\mathfrak{f}(5 \omega)^{8}-\mathfrak{f}_{1}(5 \omega)^{8}\right)+32 /|\mathfrak{f}(5 \omega)|^{8} \\
=|\mathfrak{f}(5 \omega)|^{16}-\left|\mathfrak{f}_{1}(5 \omega)\right|^{16}-\left|\mathfrak{f}_{2}(5 \omega)\right|^{16}+32 /|\mathfrak{f}(5 \omega)|^{8} .
\end{gathered}
$$

Thus, writing $l=|\mathfrak{f}(5 \omega)|^{2}, m=\left|\mathfrak{f}_{1}(5 \omega)\right|^{2} / \sqrt{2}$ and $n=\left|\mathfrak{f}_{2}(5 \omega)\right|^{2} \sqrt{2}$ so that $l m n=1$, we have

$$
16 /(m n)^{4}-64=16 m^{8}+16 n^{8}-32(m n)^{4} .
$$

Rearranging and completing the square on both sides, we obtain

$$
\left(8(m n)^{2}-4 /(m n)^{2}\right)^{2}=\left(4 m^{4}+4 n^{4}\right)^{2} .
$$

After taking the correct square root, we find

$$
1 /(m n)^{2}-2(m n)^{2}=m^{4}+n^{4},
$$

which after rearranging again and taking the correct square root gives

$$
1 /(m n)=m^{2}+n^{2}
$$

that is

$$
m^{3} n+m n^{3}=1
$$

Again we note that this equation holds more generally than for the value of $\tau$ that we chose. In fact we only require that $\tau$ is a root of a binary quadratic form with $c=5 a$.

By arguments similar to those of the previous subsection, we are able to obtain all the evaluations in the above table.

2.3. Discriminant -103. Again similar techniques work for $d=-103$, the next discriminant for class number 5 .

We evaluate the expression

$$
\begin{gathered}
m_{1}=\sqrt{2}\left|\frac{\eta((1+\sqrt{-103}) / 2)}{\eta((1+\sqrt{-103}) / 4)}\right|^{2}=\sqrt{2} /\left|\mathfrak{f}_{1}\left(\frac{1+\sqrt{-103}}{2}\right)\right|^{2} \\
=\sqrt{2} /\left|\mathfrak{f}\left(\frac{-1+\sqrt{-103}}{2}\right)\right|^{2}=\sqrt{2} /\left|\mathfrak{f}\left(\frac{1+\sqrt{-103}}{2}\right)\right|^{2} .
\end{gathered}
$$


Since both of the last expressions in the two rows of this equation have the same value, then from (4) we see that these expressions are equal to

$$
\left|\mathfrak{f}_{2}\left(\frac{1+\sqrt{-103}}{2}\right)\right|=\sqrt{2} /\left|\mathfrak{f}_{1}(1+\sqrt{-103})\right|=\sqrt{2} /|\mathfrak{f}(\sqrt{-103})| .
$$

This evaluation is strangely not made in either [18] or [1], however Weber's method works fine here, and making use of a computer to handle the $q$-series involved in computing the relevant modular equation, we find that $m_{1}$ has minimal polynomial $x^{5}+2 x^{4}+3 x^{3}+3 x^{2}+x-1$.

Now it will be convenient to write $m_{1}$ in a slightly different form. From the first equation for $m_{1}$ above we see that

$$
m_{1}=\sqrt{2} /\left|\mathfrak{f}_{1}\left(\frac{1+\sqrt{-103}}{2}\right)\right|^{2}=\left|\mathfrak{f}_{2}\left(\frac{1+\sqrt{-103}}{4}\right)\right|^{2} / \sqrt{2} .
$$

We will denote this unit by $n$, for consistency with the earlier cases we have dealt with.

Now we also wish to calculate the value $m_{2}$ defined by

$$
\begin{gathered}
1 / m_{2}=\left|\frac{\eta((3+\sqrt{-103}) / 8)}{\eta((1+\sqrt{-103}) / 4)}\right|^{2} / \sqrt{2}=\left|\frac{\eta((-3+\sqrt{-103}) / 8)}{\eta((-3+\sqrt{-103}) / 4)}\right|^{2} / \sqrt{2} \\
=\left|\mathfrak{f}_{1}\left(\frac{-3+\sqrt{-103}}{4}\right)\right|^{2} / \sqrt{2}=\left|\mathfrak{f}\left(\frac{1+\sqrt{-103}}{4}\right)\right|^{2} / \sqrt{2} .
\end{gathered}
$$

Again for consistency we denote this value by $l$. Of course we also write

$$
m=\left|\mathfrak{f}_{1}\left(\frac{1+\sqrt{-103}}{4}\right)\right|^{2} \text {, }
$$

so that $l m n=1$.

In order to make use of a modular equation we express the values $l, m$ and $n$ in terms of values with argument $\omega=\frac{3+\sqrt{-103}}{28}$. In fact by making simple transformations of the expressions above we find that $m=|\mathfrak{f}(7 \omega)|^{2}, n=\left|\mathfrak{f}_{2}(7 \omega)\right|^{2} / \sqrt{2}$ and $l=$ $\left|\mathfrak{f}_{1}(7 \omega)\right|^{2} / \sqrt{2}$.

Now we note that

$$
\mathfrak{f}(\omega)=\mathfrak{f}\left(\frac{3+\sqrt{-103}}{28}\right)=\mathfrak{f}\left(\frac{-3+\sqrt{-103}}{4}\right)=\overline{\mathfrak{f}}(7 \omega) .
$$

Similarly we find that

$$
\mathfrak{f}_{1}(\omega)=\overline{\mathfrak{f}_{2}}(7 \omega), \quad \mathfrak{f}_{2}(\omega)=\overline{\mathfrak{f}_{1}}(7 \omega) .
$$

Now the appropriate modular equation is Weber's modular equation of irrational form of degree 7 . This is given by Weber in $\S 75$ of [18] as

$$
\mathfrak{f}(\omega) \mathfrak{f}(7 \omega)-\mathfrak{f}(\omega) \mathfrak{f}(7 \omega)-\mathfrak{f}_{1}(\omega) \mathfrak{f}_{1}(7 \omega)=0,
$$

which, for the specific value of $\omega$ that we have chosen, becomes

$$
\overline{\mathfrak{f}}(7 \omega) \mathfrak{f}(7 \omega)-\overline{\mathfrak{f}_{2}}(7 \omega) \mathfrak{f}_{1}(7 \omega)-\overline{\mathfrak{f}_{1}}(7 \omega) \mathfrak{f}_{2}(7 \omega)=0 .
$$

We remove the asymmetry from this equation as we did in earlier cases and obtain

$$
\left(l^{4}+n^{4}\right)^{2}=8 / m+m^{5}-5 m^{2} .
$$

Of course we can use the expression $l m n=1$ to change this equation so that it is only in terms of two of the values $l, m$ and $n$. 


\begin{tabular}{|c|c|}
\hline Eta Evaluations $(d=-103)$ & Minimum Polynomial \\
\hline$\sqrt{2} /\left|\mathfrak{f}_{1}\left(\frac{1+\sqrt{-103}}{2}\right)\right|^{2}=\sqrt{2} /|\mathfrak{f}(\sqrt{-103})|$ & $x^{5}+2 x^{4}+3 x^{3}+3 x^{2}+x-1$ \\
$=\left|\mathfrak{f}_{2}\left(\frac{1+\sqrt{-103}}{4}\right)\right| / \sqrt{2}$ & \\
\hline$\left|\mathfrak{f}_{1}\left(\frac{3+\sqrt{-103}}{4}\right)\right|^{2} / \sqrt{2}=\left|\mathfrak{f}\left(\frac{1+\sqrt{-103}}{4}\right)\right|^{2} / \sqrt{2}$ & $x^{5}-3 x^{4}+5 x^{3}-4 x^{2}+x-1$ \\
\hline
\end{tabular}

Table 2.3

First, we subtract $4 / m^{4}=4 l^{4} m^{4}$ from each side and multiply through by $m^{4}$. After rearranging we obtain

$$
m^{4}\left(l^{4}+n^{4}\right)^{2}=\left(m^{3}-1\right)\left(m^{3}-2\right)^{2} .
$$

Dividing through by $m^{9}$ and using $1 / m=\ln$ we obtain

$$
(\ln )^{5}\left(l^{4}-n^{4}\right)^{2}=\left(1-(\ln )^{3}\right)\left(1-2(\ln )^{3}\right)^{2} .
$$

Since we already have a minimal polynomial for $n$, this equation provides us with one for $l$.

We summarise the eta evaluations obtained in Table 2.3.

2.4. Discriminant -127. Finally for class number five we look at the discriminant $d=-127$. The process for this discriminant is very similar to that for $d=-79$ so we simply summarise the steps involved.

Firstly we wish to evaluate

$$
\begin{aligned}
m_{1}=\sqrt{2}\left|\frac{\eta((1+\sqrt{-127}) / 2)}{\eta((1+\sqrt{-127}) / 4}\right|^{2} & =\sqrt{2} /\left|\mathfrak{f}_{1}\left(\frac{1+\sqrt{-127}}{2}\right)\right|^{2} \\
& =\sqrt{2} /\left|\mathfrak{f}\left(\frac{1+\sqrt{-127}}{2}\right)\right|^{2}=\sqrt{2} /|\mathfrak{f}(\sqrt{-127})| .
\end{aligned}
$$

But this is also equal to $\left|\mathfrak{f}_{2}((1+\sqrt{-127}) / 4)\right|^{2} / \sqrt{2}$.

Likewise we want $m_{2}$, given by

$$
1 / m_{2}=\left|\frac{\eta((1+\sqrt{-127}) / 8)}{\eta((1+\sqrt{-127}) / 4}\right|^{2} / \sqrt{2}=\left|\mathfrak{f}_{1}\left(\frac{1+\sqrt{-127}}{4}\right)\right|^{2} / \sqrt{2} .
$$

We call the first of these values above $n$, the second $m$ and to be consistent with what has gone before we denote

$$
l=\left|\mathfrak{f}\left(\frac{1+\sqrt{-127}}{4}\right)\right|^{2},
$$

so that as usual $\operatorname{lm} n=1$.

Again neither Weber nor Ramanujan/Berndt evaluate $|\mathfrak{f}(\sqrt{-127})|$, however Weber's method can be applied and we find that $n$ satisfies $n^{5}-n^{4}-2 n^{3}+n^{2}+3 n-1=0$.

We write

$$
m=\left|\mathfrak{f}_{1}\left(\frac{-7+\sqrt{-127}}{4}\right)\right|^{2} / \sqrt{2}, \quad n=\left|\mathfrak{f}_{2}\left(\frac{-7+\sqrt{-127}}{4}\right)\right|^{2} / \sqrt{2}, \text { etc. }
$$

Now we can make use of the modular equation of degree 11, since

$\mathfrak{f}_{2}\left(\frac{-7+\sqrt{-127}}{4}\right)=\mathfrak{f}_{1}\left(\frac{7+\sqrt{-127}}{44}\right), \mathfrak{f}_{1}\left(\frac{-7+\sqrt{-127}}{4}\right)=\mathfrak{f}_{2}\left(\frac{7+\sqrt{-127}}{44}\right)$, 


\begin{tabular}{|c|c|}
\hline Eta Evaluations $(d=-127)$ & Minimum Polynomial \\
\hline$\sqrt{2} /\left|\mathfrak{f}_{1}\left(\frac{1+\sqrt{-127}}{2}\right)\right|^{2}=\sqrt{2} /|\mathfrak{f}(\sqrt{-127})|$ & $x^{5}-x^{4}-2 x^{3}+x^{2}+3 x-1$ \\
$=\left|\mathfrak{f}_{2}\left(\frac{1+\sqrt{-127}}{4}\right)\right| / \sqrt{2}$ & \\
\hline$\left|\mathfrak{f}_{1}\left(\frac{1+\sqrt{-127}}{4}\right)\right|^{2} / \sqrt{2}$ & $x^{5}-x^{3}-4 x^{2}+4 x-1=0$ \\
\hline
\end{tabular}

Table 2.4

and so on.

In a manner similar to our previous arguments, the modular equation for degree 11 becomes

$$
\overline{\mathfrak{f}}(11 \tau)^{2} \mathfrak{f}(11 \tau)^{2}-\overline{\mathfrak{f}_{2}}(11 \tau)^{2} \mathfrak{f}_{1}(11 \tau)^{2}-\overline{\mathfrak{f}_{1}}(11 \tau)^{2} \mathfrak{f}_{2}(11 \tau)^{2}=4,
$$

for the value $\tau=(7+\sqrt{-127}) / 44$ (or indeed for any root of a binary quadratic form having $c=11 a$.

After twice putting the asymmetric part on one side and squaring, we obtain

$$
l^{6}-6 l^{4}+17 l^{2}-24+16 / l^{2}-2 / l^{4}=m^{8}+n^{8} .
$$

After rearranging we can take the square root

$$
l^{4}-3 l^{2}+4=l\left(m^{4}+n^{4}\right) .
$$

Now we replace $l$ with $1 /(m n)$, multiply through by $(m n)^{4}$ and we have

$$
4(m n)^{4}-3(m n)^{2}+1=(m n)^{3}\left(m^{4}+n^{4}\right) .
$$

By the usual method, we determine that $m$ satisfies $x^{5}-x^{3}-4 x^{2}+4 x-1=0$. Again we summarise our evaluations in Table 2.4 above.

2.5. Class Number Seven. Unfortunately our run of luck with class number five now comes to an end. The next two fundamental discriminants with this class number are $d=-131$ and $d=-179$. However the eta quotients that are associated with these discriminants are not able to be expressed in terms of Weber functions. Note that in both cases we do not have $d \equiv 1(\bmod 8)$. This will become relevant later.

It seems that we need some new sets of functions which are eta quotients similar to the Weber function but where the arguments of the numerator and denominator differ by a factor other than 2. This was essentially the program of [8] (though the results are somewhat different in character to the current ones). For now however, we move on to higher class numbers.

The first discriminant of class number seven is $d=-71$. There are now three values which we would like to evaluate

$$
\begin{aligned}
m_{1}=\sqrt{2}\left|\frac{\eta((1+\sqrt{-71}) / 2)}{\eta((1+\sqrt{-71}) / 4)}\right|^{2}=\sqrt{2} /\left|\mathfrak{f}_{1}\left(\frac{1+\sqrt{-71}}{2}\right)\right|^{2} \\
=\left|\mathfrak{f}_{2}\left(\frac{1+\sqrt{-71}}{4}\right)\right|^{2} / \sqrt{2}=\left|\mathfrak{f}_{2}\left(\frac{3+\sqrt{-71}}{4}\right)\right|^{2} / \sqrt{2}, \\
m_{2}=\sqrt{2}\left|\frac{\eta((1+\sqrt{-71}) / 4)}{\eta((3+\sqrt{-71}) / 8)}\right|^{2}=\sqrt{2} /\left|\mathfrak{f}\left(\frac{-1+\sqrt{-71}}{4}\right)\right|^{2} \\
=\sqrt{2} /\left|\mathfrak{f}\left(\frac{1+\sqrt{-71}}{4}\right)\right|^{2}=\sqrt{2} /\left|\mathfrak{f}_{1}\left(\frac{3+\sqrt{-71}}{4}\right)\right|^{2},
\end{aligned}
$$


and

$$
\begin{aligned}
& m_{3}=\sqrt{\frac{4}{3}}\left|\frac{\eta((3+\sqrt{-71}) / 8)}{\eta((1+\sqrt{-71}) / 6)}\right|^{2}=\sqrt{\frac{4}{3}}\left|\frac{\eta((-5+\sqrt{-71}) / 8)}{\eta((-5+\sqrt{-71}) / 6)}\right|^{2} \\
= & \sqrt{2}\left|\frac{\eta((5+\sqrt{-71}) / 8)}{\eta((5+\sqrt{-71}) / 16)}\right|^{2}=\sqrt{2} /\left|\mathfrak{f}_{1}\left(\frac{5+\sqrt{-71}}{8}\right)\right|^{2}=\sqrt{2} /\left|\mathfrak{f}\left(\frac{3+\sqrt{-71}}{8}\right)\right|^{2} .
\end{aligned}
$$

The first of these can be expressed in terms of $|\mathfrak{f}(\sqrt{-71})|$. We note that

$$
\left|\mathfrak{f}_{1}\left(\frac{1+\sqrt{-71}}{2}\right)\right|=\left|\mathfrak{f}\left(\frac{1+\sqrt{-71}}{2}\right)\right|,
$$

and so

$$
\left|\mathfrak{f}_{2}\left(\frac{1+\sqrt{-71}}{2}\right)\right|=\sqrt{2} /\left|\mathfrak{f}_{1}\left(\frac{1+\sqrt{-71}}{2}\right)\right|^{2}=m_{1} .
$$

But now

$$
m_{1}=\sqrt{2} /\left|\mathfrak{f}_{1}(1+\sqrt{-71})\right|=\sqrt{2} /|\mathfrak{f}(\sqrt{-71})| .
$$

But Weber has computed this last value in the tables at the end of his Lehrbuch[18]. We see in fact that $m_{1}$ has minimal polynomial $x^{7}+x^{6}-x^{5}-x^{4}-x^{3}+x^{2}+2 x-1$.

Now we relate $m_{1}$ and $m_{2}$ with a modular equation of degree 5 . We note that

$$
\mathfrak{f}_{1}\left(\frac{-3+\sqrt{-71}}{4}\right)=\mathfrak{f}_{2}\left(\frac{3+\sqrt{-71}}{20}\right), \text { etc. }
$$

We already know that the modular equation of degree five leads to the relation (12). In the present case it will relate $m_{1}$ with $1 / m_{2}$, i.e.

$$
m_{1}^{3} / m_{2}+m_{1} / m_{2}^{3}=1 \text {. }
$$

This allows us to compute that $m_{2}$ is the real root of $x^{7}-2 x^{6}+4 x^{5}-4 x^{4}+5 x^{3}-$ $4 x^{2}+2 x-1$.

Now from the definitions above, we see that $m_{3}$ is equal to

$$
\sqrt{2} /\left|\mathfrak{f}_{1}\left(\frac{5+\sqrt{-71}}{8}\right)\right|^{2}=\sqrt{2} /\left|\mathfrak{f}_{1}\left(\frac{11+\sqrt{-71}}{8}\right)\right|^{2},
$$

whilst $m_{2}$ becomes the value

$$
\sqrt{2} /\left|\mathfrak{f}_{1}\left(\frac{3+\sqrt{-71}}{4}\right)\right|^{2}=\left|\mathfrak{f}_{2}\left(\frac{3+\sqrt{-71}}{8}\right)\right|^{2} / \sqrt{2}=\left|\mathfrak{f}_{2}\left(\frac{11+\sqrt{-71}}{8}\right)\right|^{2} / \sqrt{2} .
$$

But now we note that

$$
\mathfrak{f}_{2}\left(\frac{-11+\sqrt{-71}}{8}\right)=\mathfrak{f}_{2}\left(\frac{11+\sqrt{-71}}{24}\right), \text { etc. }
$$

Therefore we can use the modular equation of degree three. We find that $m_{3}$ has minimal polynomial $x^{7}+x^{6}+2 x^{5}+2 x^{4}+x^{3}-2 x^{2}-3 x-1$.

Of course, using the modular equations that we have above, we can provide many other evaluations. However, eventually the technique runs into trouble, in that modular equations of irrational form of degrees 13, 17 and 19 were not even computed by Weber, much less can we turn them into relations of the kind which we have used above. Even for modular equations of higher degree which Weber did calculate (e.g. for the prime 23, there does not appear to be a conversion process analogous to the one we used, to obtain the required relations between class invariants). 
However, not all is lost, since a different kind of modular equation can be used to extend the method we have been discussing, in a systematic way, as we explain in the remainder of this paper.

\section{The General Method}

The method we have relied on to this point has been to convert Weber modular equations such as (8) into a 'symmetric' form, i.e. where values always occur along with their complex conjugate. As noted, this becomes difficult to achieve, if not impossible, as the Weber modular equations become more complicated.

It seems logical to attempt to switch $\mathfrak{f}_{1}(n \tau)$ and $\mathfrak{f}_{2}(n \tau)$ in the definition of these modular equations of degree $n$, and recompute them in this altered form. Then the values that would result when we specialise would automatically occur multiplied by their complex conjugate, eliminating the need to first manipulate the modular equation. However, this approach simply does not work.

As discussed in our paper [9], a slight permutation of this idea is successful, and we will show here that the new kind of modular equation computed there, does in fact lead to the symmetric relations involving class invariants, that we are after.

Specifically, instead of the functions which Weber uses, we define

$$
\begin{gathered}
C=(\mathfrak{f}(\tau) \mathfrak{f}(n \tau))^{k} \\
D=\left(\mathfrak{f}_{1}(\tau) \mathfrak{f}_{2}(n \tau)\right)^{l} \pm\left(\mathfrak{f}_{2}(\tau) \mathfrak{f}_{1}(n \tau)\right)^{l},
\end{gathered}
$$

where $n$ is the degree of the modular equation, and the sign in $D$ and the values $k, l \in \mathbb{N}$, depend on $n$.

Now we choose our value $\tau$ carefully. We suppose that

$$
\tau=\frac{b+\sqrt{d}}{2 n a} \text { and }-1 / \tau=\frac{-b+\sqrt{d}}{2 a} \text { for some } n \in \mathbb{N} .
$$

Note, this is equivalent to $\tau$ being a root of a binary quadratic form $(a, b, c)$ with $c=n a$.

When we plug this value of $\tau$ in, we obtain

$$
C=|\mathfrak{f}(n \tau)|^{2 k} \text { and } D=\left|\mathfrak{f}_{1}(n \tau)\right|^{2 l} \pm\left|\mathfrak{f}_{2}(n \tau)\right|^{2 l} .
$$

Thus, the modular equations in question, being polynomial relations between $C$ and $D$, immediately yield relations similar to the ones used in the first section of this paper.

To make use of this equation, we first write $C=\frac{2^{k}}{\left|\mathfrak{f}_{1}(n \tau) \mathfrak{f}_{2}(n \tau)\right|^{2 k}}$, and then using (4), we write $\mathfrak{f}_{2}(n \tau)=\sqrt{2} / \mathfrak{f}_{1}(2 n \tau)$. This yields a polynomial relation between

$$
C=\left|\frac{\mathfrak{f}_{1}(2 n \tau)}{\mathfrak{f}_{1}(n \tau)}\right|^{2 k} \text { and } D=\left|\mathfrak{f}_{1}(n \tau)\right|^{2 l} \pm \frac{2^{l}}{\left|\mathfrak{f}_{1}(2 n \tau)\right|^{2 l}} \text {. }
$$

In other words, we obtain a polynomial relating $\left|\mathfrak{f}_{1}(n \tau)\right|^{2}$ and $\left|\mathfrak{f}_{1}(2 n \tau)\right|^{2}$. Rewriting $n \tau$ as $\tau$, we have a polynomial relation of the form

$$
P\left(\left|\mathfrak{f}_{1}(\tau)\right|^{2},\left|\mathfrak{f}_{1}(2 \tau)\right|^{2}\right)=0 .
$$

Also, we can eliminate any one of the Weber functions of our chosing from the original polynomial expression relating $C$ and $D$, by making use of the identity $\mathfrak{f f}_{1} \mathfrak{f}_{2}=\sqrt{2}$. Again rewriting $\tau$ for $n \tau$, this leaves an identity of the form

$$
P_{i, j}\left(\left|\mathfrak{f}_{i}(\tau)\right|^{2},\left|\mathfrak{f}_{j}(\tau)\right|^{2}\right)=0,
$$


where the $i$ and $j$ signify different Weber functions.

Before we can make use of such relations, however, we must first express the eta quotients we are interested in, in terms of Weber functions.

As mentioned already, we are interested in evaluating eta quotients where the arguments are taken to be roots of binary quadratic forms of the same discriminant $d \equiv 1(\bmod 8)$. In fact, we can take one representative binary quadratic form from each class in the form class group, since all others may be obtained by modular transformations. Thus there will be a form class associated to both the numerator and denominator of each eta quotient.

Furthermore, we do not need to compute every such eta quotient. Let us somehow order the classes of the class group, $C_{0}, C_{1}, C_{2}, \ldots, C_{m}$, but exclude classes from the list which are opposite, i.e. we demand that the list does not contain a class corresponding to the form $(a, b, c)$ and also that corresponding to $(a,-b, c)$. Then it suffices to evaluate the eta quotients $\left|\eta\left(C_{0}\right) / \eta\left(C_{1}\right)\right|^{2},\left|\eta\left(C_{1}\right) / \eta\left(C_{2}\right)\right|^{2}, \ldots$, $\left|\eta\left(C_{m-1}\right) / \eta\left(C_{m}\right)\right|^{2}$ and $\left|\eta\left(C_{m}\right) / \eta\left(C_{0}\right)\right|^{2}$, since all the others may be obtained by multiplying ones of this form.

But since we are free to choose which representatives of each class we use in each case, we will make our choice in such a way that as many of these eta quotients as possible, can be expressed as Weber functions.

Note that since $d \equiv 1(\bmod 8), 2$ splits as a product of two prime ideals in $\mathbb{Q}(\sqrt{d})$, $2=\mathfrak{p p}^{\prime}$. In particular, these primes do not occur in the principal ideal class (except where the discriminant is -7 , where the class number is one and therefore in a case of no interest to us) and they have norm 2. Thus the class for which $\mathfrak{p}$ is a representative, generates a nontrivial subgroup $H$ of the ideal class group.

Translating this fact into statements about the form class group, we see that the ideal classes in $H$ correspond to form classes containing representatives whose roots differ by a factor of 2 . Thus the forms in this subgroup can be ordered in a cycle, so that the eta quotients for consecutive classes in the list are in fact Weber functions. Of course we then remove any non-distinct eta quotients arising from opposite forms.

Let us call a complete set of non-opposite form classes, a full set of classes, and a set of roots of representatives of such a full set, a full set of roots.

Now each coset of $H$ also has a set of Weber function values associated with it, by a similar argument to the above. Collecting together all the Weber function values obtained from the various cosets in this way, we end up with a collection of Weber functions $\mathfrak{f}_{1}(\tau)$ where $\tau$ ranges over a full set of roots for the given discriminant.

In fact, we cannot construct all eta quotients from these Weber function values. What is missing is the set of eta quotients with numerator and denominator in distinct cosets of $H$. These eta quotients cannot be expressed in terms of Weber functions, and we will not evaluate them with our technique. Furthermore, we will not be able to evaluate all the Weber functions themselves. We will certainly evaluate all those which are associated with classes in the subgroup $H$. Also, in certain cases we are able to evaluate all those associated with classes in various cosets of $H$.

Firstly we note that the full set of roots can continue to be thought of as organised into subsets corresponding to the original cosets of $H$. Each such subset is also ordered so that adjacent roots come from classes which were adjacent in the above argument. In particular, any two adjacent roots can be transformed by modular substitutions so that they differ by a factor of 2 . 
However in applying a modular transformation to a root $\tau,\left|\mathfrak{f}_{1}(\tau)\right|^{2}$ may become $\left|\mathfrak{f}\left(\tau^{\prime}\right)\right|^{2},\left|\mathfrak{f}_{1}\left(\tau^{\prime}\right)\right|^{2}$ or $\left|\mathfrak{f}_{2}\left(\tau^{\prime}\right)\right|^{2}$ for some $\tau^{\prime}$ in the same class as $\tau$. Thus two adjacent Weber functions may become $\left|\mathfrak{f}_{i}\left(\tau^{\prime}\right)\right|^{2}$ and $\left|\mathfrak{f}_{j}\left(2 \tau^{\prime}\right)\right|^{2}$, where the $i$ and $j$ signify that we may have any of the three Weber functions. Let us suppose this has been done, and call the new $\tau^{\prime}, \tau$ again, to save on notation.

We now have two problems to overcome before we can use the relations above, derived from modular equations, to relate these adjacent values. First the relations above are only valid if the $\tau$ that we end up with is a root of a form $(a, b, c)$ with $c=n a$ for some $n \in \mathbb{N}$. Secondly, we need to show how to use the relations we have available to provide relations between all the different combinations of Weber functions that are possible.

The first problem is overcome by applying transformations to the value $\tau$ and corresponding transformations to $2 \tau$ so that they are both of the required form. Suppose that $\tau$ is a root of the form $(2 a, b, c)$, so that $2 \tau$ is a root of $(a, b, 2 c)$.

If we add $k$ to the value $\tau$ and $2 k$ to the value $2 \tau$, the new forms that these values correspond to are $\left(2 a, b+4 k a, c+2 k^{2} a+b k\right)$ and $\left(a, b+4 k a, 2 c+4 k^{2} a+2 k b\right)$. Of course if $(a, b)=1$, we can always choose $k$ such that $2 a \mid c+2 k^{2} a+b k$ and thus also $a \mid 2 c+4 k^{2} a+2 k b$. Note that the new roots that we obtain after this transformation are still in the ratio $1: 2$.

If $(a, b)=s>1$, we first note that since $d=b^{2}-4 a c$ we must have $s \mid d$, so that there are only a finite number of primes to be concerned with. A similar thing is true if $(c, b)=s^{\prime}>1$. Thus to deal with this problem, we first apply the transformation $\tau \rightarrow-1 / \tau$ to both $\tau$ and $2 \tau$. This has the effect of changing the associated forms to $(c,-b, 2 a)$ and $(2 c,-b, a)$. We then add $2 k$ to the first value and $k$ to the second for a carefully chosen $k$ such that we obtain new forms $\left(c, b^{\prime}, 2 a^{\prime}\right)$ and $\left(2 c, b^{\prime}, a^{\prime}\right)$ with $\left(b^{\prime}, a^{\prime}\right)=1$. We again apply $\tau \rightarrow-1 / \tau$ to both values and obtain forms $\left(2 a^{\prime}, b^{\prime}, c\right)$ and $\left(a^{\prime}, b^{\prime}, 2 c\right)$. To these we apply the method of the proceeding paragraph.

In all cases, we see that we are able to transform the values $\tau$ and $2 \tau$ so that they are roots of forms $(2 a, b, c)$ and $(a, b, 2 c)$ with $2 a \mid c$ and of course $a \mid 2 c$. Thus in both cases the identities we wish to use are valid for $\tau$ and $2 \tau$.

But now because of all this, we can relate $\left|\mathfrak{f}_{i}(\tau)\right|^{2}$ with $\left|\mathfrak{f}_{1}(\tau)\right|^{2}$ using a relation of the form (15), then relate $\left|\mathfrak{f}_{1}(\tau)\right|^{2}$ to $\left|\mathfrak{f}_{1}(2 \tau)\right|^{2}$ with $(14)$, then relate $\left|\mathfrak{f}_{1}(2 \tau)\right|^{2}$ with $\left|\mathfrak{f}_{j}(2 \tau)\right|^{2}$ with a relation of the form (15).

Thus we have established that all Weber function values corresponding to a given coset can be related to each other using the identities we derived from modular equations.

The only thing which remains is for us to actually evaluate one of the Weber function values in some of these subsets. Then because of all the relations we have, we will be able to evaluate all the Weber functions in those subsets.

The one case where we can always directly evaluate one of the Weber functions in a subset is that of the subset associated to the principal class. In particular we consider a Weber function evaluated at the root $\frac{1+\sqrt{d}}{2}$ of the form $(1,1,(1-d) / 4)$. To evaluate this, we note

$$
\left|\mathfrak{f}_{1}\left(\frac{1+\sqrt{d}}{2}\right)\right|^{2}=\left|\mathfrak{f}\left(\frac{-1+\sqrt{d}}{2}\right)\right|^{2}=\left|\mathfrak{f}\left(\frac{1+\sqrt{d}}{2}\right)\right|^{2} .
$$

Thus, making use of $\mathfrak{f f}_{1} \mathfrak{f}_{2}=\sqrt{2}$, we find that we only need to evaluate $\left|\mathfrak{f}_{2}\left(\frac{1+\sqrt{d}}{2}\right)\right|$. However, by (4) this is equivalent to evaluating $\left|\mathfrak{f}_{1}(1+\sqrt{d})\right|=|\mathfrak{f}(\sqrt{d})|$. 
These are values which can be computed using existing methods, e.g: by the methods employed by Weber as described in the first section of this paper.

However, there are various other instances where we can evaluate one of the Weber functions in the subsets associated to cosets of $H$. For it may be that the root $\frac{k+\sqrt{d}}{2 k}$ is a root of a form of discriminant $d$, which is associated with one of these other subsets.

The form this root corresponds to is $(k, k, c)$ for some $c$. But this implies $k^{2}-4 k c=$ $d$. Clearly then, $k \mid d$, i.e. $\mathrm{d}=\mathrm{kk}$. Then we only require $k-4 c=k^{\prime}$ for the form to have discriminant $d$. Thus this situation arises when $d$ can be split into two factors which are the same modulo 4 . However, this is automatic, since $d \equiv 1(\bmod 8)$, and therefore a form of the kind described arises for each factor $k$ of $d$.

However in such cases, we see that

$$
\left|\mathfrak{f}_{1}\left(\frac{k+\sqrt{d}}{2 k}\right)\right|^{2}=\left|\mathfrak{f}\left(\frac{-k+\sqrt{d}}{2 k}\right)\right|^{2}=\left|\mathfrak{f}\left(\frac{k+\sqrt{d}}{2 k}\right)\right|^{2} \text {. }
$$

Thus, evaluating $\left|\mathfrak{f}_{1}\left(\frac{k+\sqrt{d}}{2 k}\right)\right|^{2}$ is equivalent to evaluating $\left|\mathfrak{f}_{2}\left(\frac{k+\sqrt{d}}{2 k}\right)\right|$ which, as above, boils down to evaluating $\sqrt{2} /\left|\mathfrak{f}_{1}\left(\frac{k+\sqrt{d}}{k}\right)\right|=\left|\mathfrak{f}\left(\frac{\sqrt{d}}{k}\right)\right|$. However, it is possible to relate this last value to $|\mathfrak{f}(\sqrt{d})|$ by means of a Schläfli modular equation. These are described in [18] and also for composite degree, in [8].

In the final section of this paper, we will give some explicit computations demonstrating the method we have described, completing evaluations associated both with the subset corresponding to the subgroup $H$, generated by the prime over 2 , and at values associated with cosets of this subgroup, as just outlined.

\section{REFERENCES}

[1] Bruce Berndt, Ramanujan's Notebooks Part V Springer-Verlag, 1998

[2] Bruce C. Berndt, Heng Huat Chan, Liang-Cheng Zhang, Ramanujan's class invariants and cubic continued fraction. Acta Arith. 73, No.1, (1995), pp. 67-85.

[3] Bruce C. Berndt, Heng Huat Chan, Liang-Cheng Zhang, Ramanujan's class invariants, Kronecker's limit formula, and modular equations. Trans. Am. Math. Soc. 349, No.6, (1997), pp. 2125-2173.

[4] B. J. Birch Weber's class invariants Mathematika 16 (1969), pp. 283-294.

[5] Robin Chapman, William B. Hart Evaluation of the Dedekind eta function To appear in the Canadian Mathematical Bulletin.

[6] A. Gee Class invariants from Dedekind's eta function Thesis, Thomas Stieltjes Institute for Mathematics, 2001

[7] F. Hajir Elliptic units of cyclic unramified extensions of complex quadratic fields Acta Arith. LXIV.1 (1993) pp. $69-85$.

[8] William Hart, Schlaefli modular equations for generalized Weber functions To appear in the Ramanujan Journal.

[9] William Hart, A new class of Weber-like modular equations In preparation.

[10] W. B. Hart Explicit evaluation of the Dedekind eta function PhD. Thesis, 2004, Macquarie University, Sydney.

[11] H. Muzaffar, K. S. Williams Evaluation of Weber's functions at quadratic irrationalities JP Jour. Algebra, Number Theory and Appl., 4(2), (2004), pp. 209-259.

[12] G. N. Watson Some singular moduli. I. Q. J. Math., Oxf. Ser. 3, (1932), pp. 81-98.

[13] " " " "Some singular moduli. II. Q. J. Math., Oxf. Ser. 3, (1932), pp. 189-212.

[14] " " " "Singular moduli. III. Proc. Lond. Math. Soc., II. Ser. 40, (1935), pp. 83-142.

[15] " " " "Singular moduli IV. Acta Arith. 1, (1936) pp. 284-323.

[16] " " " "Singular moduli. V. Proc. Lond. Math. Soc., II. Ser. 42, (1937), pp. 377-397.

[17] " " " "Singular moduli. VI. Proc. Lond. Math. Soc., II. Ser. 42, (1937), pp. 398-409.

[18] Heinrich Weber, Lehrbuch der Algebra Dritter Band, Third Edition. Chelsea N.Y.

E-mail address: wbhart@math.leidenuniv.nl 